\title{
Performance: entre el arte, la identidad, la vida y la muerte*
}

Rosa Maria Blanca**

\section{Resumo}

El presente artículo discute diferentes prácticas artísticas de la contemporaneidad que utilizan el lenguaje del performance, para problematizar la estética de la identidad. El objetivo es estudiar el aspecto gestual y visual del proceso en que se desarrolla el performance, en niveles más amplios que el de su ejecución, cómo lo es su documentación como dispositivo performativo. Se sugiere que el performance provoca una tensión en el orden hegemónico social - binario, en contextos de asimetrías y violencias de género. Son analizadas prácticas artísticas trans y del activismo latinoamericano, bajo una perspectiva queer (Rosa Blanca, 2011) y decolonial (2004).

Palavras-chave: Arte Contemporáneo, Identidades, Performance, Perspectiva Queer, Procesos de Subjetivación.

* Recebido para publicação em 9 de setembro de 2014, aceito em 25 de novembro de 2015.

** Professora no Curso de Artes Visuais e no Programa de Pós-Graduação em Artes Visuais, do Departamento de Artes Visuais, da Universidade Federal de Santa Maria, Santa Maria, RS, Brasil. rosablanca.art@gmail.com 
440 Performance: entre el arte, la identidad, la vida y la muerte

Performance: Between Art, Identity, Life and Death

\begin{abstract}
This article discusses contemporary artistic practices that use the aesthetics of identity. The aim is to study the gestural and visual process in which the performance takes place in the broader levels of implementation, how is the documentation as performative device. It is suggested that the performance causes a strain on the social hegemonic order - binary, in contexts of asymmetries and gender violence. Artistic practices trans and from Latin American activism are analyzed, under a queer perspective (Rosa Blanca, 2011) and decolonial (Walter Mignolo, 2003).
\end{abstract}

Key Words: Contemporary Art, Identity, Performance, Queer Perspective, Subjectivities. 


\section{Introducción}

El presente artículo discute diferentes prácticas artísticas de la contemporaneidad que utilizan el lenguaje del performance para problematizar la estética de la identidad. Se pretende problematizar la noción de performance de forma inter y multidisciplinar, estableciendo un diálogo entre distintas áreas del conocimiento como los estudios de género y el arte contemporáneo. El objetivo es estudiar el aspecto gestual y visual del proceso en que se desarrolla el performance, en niveles más amplios que el de su ejecución, cómo lo es su documentación.

Es así como la investigación se insiere en el contexto de la documentación visual. La idea de documentación visual como dispositivo, surge como una forma de producir conocimiento a partir del arte contemporáneo. A este dispositivo que trabaja con acciones de documentación lo denomino Centro de Documentación Electrónica $(\mathrm{CDE})^{1}$. Se trata de un centro que funciona como un proyecto continuado para idealizar y realizar acciones artísticas y culturales, empleando nociones de otros campos como los estudios de género, los estudios queer y la antropología. Se propone la interpretación de las prácticas artísticas de tal forma que los objetos electrónicos que se produzcan, como el vídeo, puedan ser utilizados como herramientas de aprendizaje (Glowczewski s/d). También, se dispone el $\mathrm{CDE}$ como un dispositivo para su interacción y, de esa manera, producir conocimiento, investigando blogs, sites y otras experiencias en performance, que serán discutidas a lo largo del artículo. Dada su capacidad de persistencia, el sistema de documentación, como modo de operar conocimiento, supera los performances en vivo (Taylor, 2011).

Se propone a la documentación como un acto performático que, más allá de la búsqueda de la traducción de los eventos artísticos, utiliza a la tecnología como un dispositivo visual

1 Actual y temporalmente, el CDE está vinculado a la Pinacoteca de la Universidad Feevale, en Novo Hamburgo. 
atravesador de procesos de conocimiento y de procesos de subjetivación. Es así como a través de acciones de documentación se proponen diversas formas de pensar lo cultural, lo artístico y lo queer en las Américas.

Se parte de dos supuestos. En el primer supuesto, se sugiere que la noción de performance en arte, coloca en tensión lo social (Blanca, 2013). En este caso, no se está refiriendo a prácticas artísticas que usan el performance como un modo de entretenimiento, como es el caso de los performance de Carmelita Tropicana o Vaginal Davis, porque más que arte, ese tipo de eventos tienen como objetivo la diversión y usan como lenguaje el escenario, el vestuario y varios otros elementos del teatro. $\mathrm{O}$ sea, en esta investigación, no es usado el performance como un momento cultural que tiene la función de equilibrar lo social. No cabe duda de que en diferentes capítulos de la historia del arte el performance ha sido desarrollado en articulación con el teatro o bien, como un espectáculo. Sólo que, tomando en cuenta los últimos sucesos políticos y culturales de las Américas, que es donde se localizan las prácticas artísticas a analizar, es posible levantar un conflicto que prevalece y da como origen manifestaciones performáticas que, con mucho, distan de formas estáticas de realización y apreciación artística. Las estéticas que se producen en los performances que trabajan los conflictos políticos identitarios, no están determinadas por las continuidades y periodicidades comunes a toda la historia del arte occidental.

En el segundo supuesto, se sugiere que la dimensión artística permite entender la visualidad más allá de la discursividad, tornando manifiestas las sensibilidades como potencialidades estéticas de los/as sujetos artistas. Lo que puede llegar a problematizar el estatuto de la identidad de género y/o artística, al estar en juego la identidad del/la propio/a artista que per forma. O sea, el/la performer produce potencias, tanto significacionales, cuanto afectivas y libertarias que lo (des)representan. Eso es arte.

Actualmente, se cuentan con centenas de publicaciones sobre performance. En lo que se refiere a obras sobre arte $y$ 
performance, publicadas o de acceso en Brasil, destaca $A$ arte da performance, de Jorge Glusberg (2007). El autor analiza el performance a través de la historia del arte, de la semiótica, de la lingüística aplicada, del psicoanálisis y del arte contemporáneo. Para Glusberg, el arte del performance obedece a una deconstrucción del cuerpo que, de alguna manera o de otra, ha estado fetichizado por la pintura, la escultura o por la literatura. Dada su fisicalidad y su relación directa con el espacio y cuestiones sociales, el performance libera a las artes del ilusionismo. En lo que se refiere al uso del cuerpo, para Glusberg, surge una forma de expresión corporal fuera de lo común a través del performance. Hay un reencuentro por parte del artista consigo mismo, una "inmanencia del gesto" (Glusberg, 2007:52).

Otra publicación que también se ha tornado referencia para los estudios sobre performance, en Brasil, es $A$ arte da performance: Do futurismo ao presente, de RoseLee Goldberg (2006). Para Goldberg, el performance es un recurso utilizado por los artistas en momentos de impasse, con la intención de cuestionar categorías y lenguajes. Describe las formas en que se ha usado el performance en el siglo XX, a partir de las vanguardias artísticas como el futurismo hasta llegar a las manifestaciones de los setentas, momento en que el performance pasa a existir como género artístico. En la medida en que se trata de una forma artística sin límites, para la autora, cada artista tiene su propia definición de performance, así como su proceso de realización. Una de las diferencias que Goldberg encuentra entre el performance del teatro y aquel que es practicado en las artes visuales, es la de que en el teatro el autor del performance es el personaje, mientras que en las artes se trata de que es el propio artista quien actúa como performer. Lo que Goldberg nos intenta indicar es que no se trata de una ficción, por definición, en el caso del performance en las artes visuales. Es la misma persona. Inclusive, Goldberg llega a explicar que, para ella, es preferible utilizar la categoría de live art, en lugar de performance ya que es un arte en vivo, cuya práctica sólo tiene sentido en su percepción directa, en el lugar y en el tiempo donde se ejecuta la acción. 
Otra publicación que debe ser tomada en cuenta, en materia de artículos científicos, es A fotografía como documento primário e performance nas artes visuais, de Regina Melim, una discusión actual sobre el estatuto de la fotografía en el proceso del performance. Al contrario de lo que Goldberg afirma, Melim se posiciona a favor de su registro. Junto con Peggy Phelan (1993), Melim considera la documentación fotográfica o videográfica como elemento integrante del performance. Mediante el uso de categorías como performativo de J. L. Austin (), Melim discurre el carácter constitutivo de la fotografía, más allá de su papel como fuente secundaria durante el registro de la acción performática. Para la autora, la fotografía como documentación, es el resultado del performance, en la medida en que intermedia el evento y la audiencia. Esto quiere decir que para Melim, las fotografías no únicamente describen el performance, sino que también lo producen en su documentación. Las fotografías serian análogas a las declaraciones performativas en el contexto de la filosofía analítica.

Douglas Negrisolli, en su publicación O corpo do performer nas artes visuais, también considera que limitar el performance al momento de su ejecución, sería excluir aquellas propuestas en que el/la artista se vale de la tecnología, como el video, como resultado final de su obra. Investiga el cuerpo del performer como soporte.

En otras áreas del conocimiento, fuera del campo del arte, la noción de performance se refiere a un acto de comunicación, cuyo estudio occidentalmente también se le ha limitado a forma/contenido. En esos dominios, los modos más eficaces de expresión son más importantes que el contenido del mensaje.

En la antropología, para Langdon (2007), el performance se caracteriza por una situación donde la función poética es dominante y cuyos resultados aparecen en la invocación de la experiencia. Lo que distingue el análisis del ritual de los estudios de performance es un movimiento direccionado en el mirar, los acontecimientos continúan siendo los mismos (2007).

En la primera parte del presente artículo, se estudian dentro de la historia de las artes como la del teatro, la pintura y la 
literatura, prácticas artísticas pioneras en el performance como una manera de producción de subjetividades e identidades artísticas capaces de atravesar el binarismo de género. Se hace énfasis en el contexto no-occidental sin dejar de discutir aquellas prácticas referenciales para la problematización identitaria.

En la segunda parte, se acentúan los aspectos de las prácticas artísticas de la contemporaneidad, prestando atención a aquellos performances que surgen en movimientos que no ignoran las condiciones políticas actuales de las Américas, como las violencias de género y los feminicidios, teniendo como inflexión el sinuoso performance de un arte en conflicto. Se recuperan las prácticas artísticas que intentan escapar de la inmovilidad de categorías como chamán, justamente, en el ámbito de lo artístico, como las realizadas por los travestis de Perú. Se complica el estatuto de la estética y del arte contemporáneo en el momento en que se menciona la participación de Giuseppe Campuzzano, del Museu de Trasvestistas del Perú, en la $31^{\text {a }}$ Bienal de Artes de São Paulo, de 2014.

Finalmente, las consideraciones.

\section{Performance e (in)corporaciones identitarias}

Mediante investigaciones recientes, se sabe que de alguna manera o de otra, el lenguaje del performance es practicado como acción estética desde hace más de ocho siglos. Sobre todo si son consideradas diversas manifestaciones culturales. En el drama popular indígena prehispánico, por ejemplo, se interactúa con un arte efímero y en movimiento donde los cuerpos de las personas que actúan se transforman a través del ritmo, los gestos, la voz y la proxémica. Es el caso de obras como el Rabinal Achí, traducida como Danza del Tun, de origen maya y que data del siglo XIII $\mathrm{d}$. C. La dinámica que provoca ese tipo de obras constituye un obstáculo para la colonialidad del poder del siglo XV. Tan es así, que la implantación de la escrita o la palabra, como forma oficial de conservación de la memoria o de construcción de la historia de la América Indígena, legitimará la censura o el apagamiento de las 
dimensiones corporales de esos montajes estéticos. La reducción de lo performático a lo gráfico, disciplinará las artes y hasta lo que ha sido denominado como "sistemas de interacción humana" (Mignolo, 2003).

En Rabinal Achí, es importante la técnica del despojo, que va desde la pérdida de órganos hasta transformaciones identitarias donde el territorio, como espacio para la invocación de fuerzas ambientales y del imaginario, se (re)constituye durante el evento estético. Quien lee las traducciones que se hicieron de esas obras, debe saber imaginar entre líneas los momentos en que se alude al sacrificio humano como una forma de evitar la desestabilización del territorio. Las traducciones al castellano van a editar esos momentos de sacrificio, eludiendo el peligro de extinción al que someten la cultura mesoamericana los españoles.

En otras obras que surgen precisamente en el siglo XVI, como la náhuatl El Bailete del Güegüense o Macho Ratón, el personaje que se manifiesta de forma irreverente desafía a la autoridad colonial (Henriquez, 2009).

En el 1600, en el kabuki japonés, la forma en que eran sugeridos los movimientos de los personajes masculinos $y$ femeninos, durante danzas dramáticas, es también considerada un modo de performance. Parece ser que o kabuki nace como un modo de protesta contra la nueva era dictatorial Tukugawa. Los estilos de vida que propone no pertenecen ni al pasado ni al presente. Es un modo de vida anticonformista, sensual y excéntrico (Martínez, 2004). Las primeras interpretaciones de crossdresser o travestismo en las artes datan de aquella época, precisamente, dentro del performance kabuki. Los actores, en su mayoría eran hombres que representaban la femineidad kabuki y debían también personificar esa identidad en su vida privada, de modo a perfeccionar su papel (2004).

Sin embargo, también se cuentan con registros anteriores al kabuki. Christine de Pizan encuentra en el performance del travestismo una forma de desarrollarse como sujeto, a propósito de una edad medieval de carácter misógina. Pizan es considerada como una de las primeras escritoras que fue capaz de construir 
autonomía cultural y financiera. Nacida hacia el final del siglo XIV y fallecida en la primera mitad del siglo $\mathrm{XV}$, Pizan es autora de obras catalogadas como obras primas pioneras del feminismo, como puede ser apreciado en Ciudad de damas (1405). Las destinatarias que Pizan escoge para sus producciones textuales son las mujeres.

En el arte de la literatura de Pizan, cada protagonista descubre sus pensamientos más íntimos, al unísono y deliberadamente se metamorfosea en el vestir y en el peinado, transitando como persona masculina, pero manteniendo la belleza intacta) (Dor, 2006. A través del performance surge una estética de lo ambiguo, de lo transitorio, precisamente en ese tránsito, en ese ir y venir entre lo femenino y lo masculino. las protagonistas triunfan. El resultado es una retórica que arguye la necesidad humana de performance, como una manera de potencializar agencias que modifiquen la condición marcadora y castrante de género. El derecho a la opción de géneros es lo que significa transgenerizarse en Pizan. Es importante esclarecer que en la primera parte de la obra, la autora, como narradora, se revuelta contra el sexo que ha recibido en el momento de su nacimiento. Esto lo podemos saber porque luego del inicio ofrece una lectura desesperada de Las lamentaciones de Mateolo, famoso compendio que naturaliza la idea de mujer con el objetivo de tornarla inferior, ampliamente distribuido en Europa, en el siglo XIV. No obstante, inmediatamente aparecerán personajes como Justicia, Derecho y Razón, quienes exhortarán a la "lectora" a construir una fortaleza y donde las piedras de esa fortificación serán mujeres guerreras y heroínas. Parece ser que es el personaje transgénero medieval de Jeanne d'Arc, célebre soldada francés, quien inspira a Pizan a creer en la fuerza de la transfiguración de la "usurpación vestimentaria". Escrito en 1429, bajo el título de Ditié de Jehanne d'Arc, el poema enfatiza, mediante la mano y puño de Pizan, la belleza de la vida del soldado Jeanne que culmina liberando a la Francia. ¿De cuál liberación habla Pizan? ¿De Francia de Jehanne o de ella misma? 
El punto es que la autora muestra cómo es abandonado el deseo de virilización, apareciendo como solución la masculinización, lo que no excluye en ese proceso de transformación, "la sensación de haber devenido otra físicamente" (Dor, 2006:11). Durante la época medieval, han sido los hombres quienes han detentado el poder de la producción bibliográfica. La misoginia ha sido intensificada por intermediación de la literatura. La voz de Pizan vulnera el poder de la literatura medieval, una época donde sólo los hombres producen las reglas del género mediante la escrita. La producción de los géneros era controlado con la mano. Pizan incursiona en el poder del lenguaje, literario.

Hasta aquí, puede ser visto cómo el performance no sólo trabaja en una dimensión de anarquismo o contestación política sino que también, usa la estética para atravesar los gestos corporales, invocando posibles lenguajes e identidades que, hasta ese momento, parecían no existir en la persona del o de la artista, pero que eternamente se expresan en devir.

En lo que se refiere a las artes visuales, la Alegoría de la Pintura (1658), realizada por Elisabetta Sirani, discute la identidad artística a través de la performance de su autorretrato (Rocco, 2006). En esa pintura, Sirani moviliza su estatus como artista femenina al performar bajo una identidad ambigua. Emerge lo autobiográfico, la imagen de artista mujer como poeta laureada, custodiada por Minerva, la diosa de las artes. Sirani se apropia de elementos identificados como propios del universo artístico y cultural masculinos, tales como el traje formal y el uso del pincel. En el Renacimiento, se dudaba de la aptitud de las mujeres para pintar. Que Sirani performe pintando, significa una evidencia visual de una acción concreta que atraviesa el discurso misógino de la época, recurriendo a una figura como la de Horacio, mediante el laurel que usa en su cabeza. Se sabe que el concepto ut picture poesis, pertenece a Horacio, expresión publicada en su Arte Poética (c. 20 a. C.) que traduce pintura como poesía, o sea, poesía silenciosa. ¿Que no es el performance el arte visible de la poesía silenciosa? ¿Esa expresión de lo que está callado, pero que 
también actúa? Entre documento y ficción, la obra de Sirani abre otros modos de pensar el estatuto de la pintura y el arte. Es evidente que en este trabajo hay un cruzamiento entre pintura, performance y género. El gesto de Sirani confirma la idea de identidad de género como performance, como una temporalidad social constituida, un resultado performativo (Butler, 2010). Es precisamente por su carácter performativo que en la repetición o ruptura subversiva existe una posibilidad de transformación de género (2010).

Hasta aquí, se ha analizado el surgimiento de un lenguaje performance en distintos contextos artísticos y temporales. Como categoría o clase independiente, el performance emerge en el contexto artístico en los años setenta (Glusberg, 2007).

En un performance no existe un guión. Pueden ser presentadas secuencias ideadas con anticipación, pero las mismas pueden mudar de acuerdo a los distintos lugares en que se presenta. Su espacio es indeterminado.

Adrien Piper es pionera en la experimentación del performance, realizando distintas experiencias con la finalidad de problematizar la idea de identidad y racialización. Estas experimentaciones con el lenguaje tratan la noción de peformance como una categoría que ha permitido que el campo del arte se abra rumbo a la interdisciplinaridad (Blanca, 2011). A partir de los sesenta, el arte ha tenido como fin la abertura de fronteras disciplinares mediante el uso de distintos medios visuales y comunicacionales. Se ha llegado a afirmar que el performance ha sido uno de los grandes desarrollos intelectuales significativos del arte en los últimos 30 años (Soussloff \& Franko, 2002). Evidentemente, están siendo considerados aquellos lenguajes que se han desdoblado a partir del performance, como los happenings, el body art y todos aquellos que problematizan el espacio artístico y cultural, incidiendo en contextos políticos, económicos, identitarios, de género y de sexualidad. Es el caso del lenguaje en vídeo, a partir del registro de performances que ha sido ampliamente explorado por artistas mujeres o lésbicas, como Lynda Benglis, en su propio trabajo Female Sensibility (1973). 
Inclusive, el registro fotográfico o videográfico es lo que ha llevado a colocar en cuestión el objeto de estudio en el arte. Esa indecibilidad de su objeto se ha traducido, en el campo del arte, en una indefinición interdisciplinar (Soussloff \& Franko, 2002). Destacan también los performances que cuestionan la identidad femenina de Martha Wilson y Jackie Apple, como Transformación: Claudia (1973), o también, el trabajo de Joan Jonas intitulado Organic Honey (1972) y, el de Eleanor Antin, bajo el título de Entalhe: Uma escultura tradicional (1972), entre otros.

Complicando los estudios en performance

En el arte contemporáneo, antes o después, el performance es un medio que el artista utiliza con claros objetivos disidentes y cuya base es anárquica (Goldberg, 2006). Es el caso de una de las propuestas que se ha decidido investigar para el presente artículo. Se trata del performance que viene realizando Manuel Mendive. Este artista cubano realiza acciones en diferentes lenguajes, como pintura, música y otras dimensiones corporales. En su obra Cuando usted cierra los ojos (1992), dialoga con la Santería Cubana. Como es sabido, la abolición de la esclavitud en Cuba data de 1886. El arte contemporáneo trabaja, en una de sus interfaces, con los estudios de diáspora. La Santería ha sido perseguida antes y después de la independencia cubana. Hay un conflicto racial, marcado por la óptica del performance. En el presente, la Santería es parte del folclore cubano.

Es de preguntarse cómo es posible que el trabajo de Mendive pueda llegar hasta nuestros días. En nuestra historia reciente, la noción de performance actualiza las prácticas artísticas marginalizadas, pero también, las puede llegar a homogeneizar, especialmente cuando son abordados por los estudios de performance. Se sabe que los estudios de performance surgen en la cultura anglo-sajona, lejos de realidades post-coloniales. $\mathrm{O}$ sea, no es lo mismo hablar sobre los performances de Yves Kline, Marina Abramovic y Joseph Beuys, que de los performances de 
Guillermo Gómez-Peña que, cuestiona la colonialidad del poder (Mignolo, 2003).

Los denominados estudios de performance estadunidenses, así como los estudios queer, tanto estadounidenses como españoles y franceses, ignoran el paradigma de la abolición. En este sentido, es posible problematizar la noción de performance como una categoría que busca la homogeneización de las prácticas artísticas que cuestionan a la identidad, ignorando el conflicto que se establece en un contexto post-abolicionista o de post-conquista. Epistemológicamente hablando, la lógica del pensamiento occidental disuelve el conflicto post-abolicionista en países de las Américas, como Cuba o Brasil.

El performance arrastra conflictos que cuestionan los sistemas políticos hegemónicos, atraviesa siglos de marcación identitaria binaria, nacional, racial y de toda la historia del colonialismo científico en las Américas, las Áfricas y las Oceanías.

El colonialismo científico se constituye en distintas áreas del conocimiento. Dentro de la antropología clásica, en los estudios de Saladin d'Auglure (2006), por ejemplo, es posible depararse con la forma en que se han categorizado determinadas personas que actúan fuera del código binario masculino/femenino - en lo que se refiere a lo que el autor denomina como hombre travesti, durante sus estudios antropológicos efectuados en la isla de Saint-Laurent, en el estrecho de Bering, Alaska. En ese tipo de investigaciones son instituidos cuerpos de análisis que son mediados visualmente. Hay una visualidad occidental que mediatiza el campo de percepción y, en consecuencia, los modos de conceptualización del antropólogo occidental. Eso quiere decir que el uso de categorías está condicionado por una visualización aprendida. El lenguaje de la antropología ha actuado como un instrumento de legitimación de identidades dicotómicas, a partir de una perspectiva de crítica postcolonial (Cunha, 2002). Haciendo uso de transcripciones etnográficas, Saladin d'Auglure enfatiza que ha prestado atención a la importancia del vestir antes que a la forma cómo actúan los personajes que etnógrafa atrás de una lente visual, formal. A través de su percepción visual, narra cómo un 
hombre con bigote se viste como mujer, advirtiendo que existe una grande consideración por este tipo de personaje que no puede ir al encuentro "de su naturaleza" (2006:302). Es obvio que D'Aglure pretende naturalizar la identidad de género mediante su descripción etnográfica, en una clasificación binaria. Esta narrativa corresponde a una de las manifestaciones de ansiedad colonial (Preciado, 2010). Es una colonización científica para enfatizar prácticas culturales del Nuevo Mundo, como África, América u Oceanía, con la finalidad de establecer categorías de género y sexualidad. La idea de travesti simplifica la complejidad del acto de interactuar con más de un género.

A menudo se ha intentado utilizar otros conceptos que ocultan el conflicto identitario de estas personas clasificadas como travestis. Se ha optado por la categoría chamán, por ejemplo. Es posible percibir que en algunos campos del conocimiento, se tiende a usar y clasificar identidades o agentes de la cultura con categorías que acompañan el modelo de pensamiento vigente occidental, como el uso de conceptos como chamán, un concepto dialéctico que reestructura el pensamiento dicotómico. Con categorías como chamán, la ciencia occidental encuentra, mediante el lenguaje, un instrumento científico y político para eliminar posibilidades identitarias que rebasan el sistema de identificación de sexo y género, de persona y cosmos, de hombre y mujer, de lo masculino y de lo femenino, de lo humano y de lo animal [Con seguridad, actualmente se han "positivado" las prácticas chamán intentando instaurar o restaurar paradigmas de una cosmogonía efectiva en el campo de la salud, denominándose como neochamanismo (Carrillo, 2003; Llamazares, 2013)].

Otro caso que subsiste hasta nuestros días, está relacionado con las trabajadoras sexuales travestidas del Perú, por ejemplo. Ellas/os se dedican al trabajo sexual, rehusando dedicarse al trabajo industrial. Ellas/os atraviesan los géneros, dando lugar a otros géneros. El travestismo en el Perú es una forma de resistencia arrastrando formas de violencia de género durante la conquista. Es así cómo surge el Museo Travestista del Perú, a cargo de Giuseppe Campuzzano, haciendo uso de 
documentaciones y exposiciones, al mismo tiempo que celebra una cultura trans contemporánea. El principal objetivo es afirmar una identidad trans, al mismo tiempo en que la complica. Lo cierto es que el Museo Travesti del Perú, como documenta-acción, es una iniciativa de reactivación de expresividades que arrastran identidades chamanísticas y no-binarias ocluidas hasta nuestros días.

La Bienal de Arte de São Paulo de 2014 ha incluido obras de Campuzano, además de otros trabajos de clara perspectiva queer y de carácter militante y activista, por primera vez en más de 30 ediciones de bienal. La crítica de arte Daniela Labra, se ha valido de convenciones ortodoxas para descalificar el trabajo de la curaduría en general de esta bienal internacional. Para Labra, al optar por trabajos políticos, Charles Esche, el curador de la Bienal de São Paulo, busca criterios menos estéticos y poco interesantes plástica y visualmente (Labra, 2014).

Es evidente que la Bienal de Arte de São Paulo de 2014, muestra una estética decolonial e insurgente. Se trata de estéticas que cruzan los espacios religiosos y políticos respondiendo al orden social heteronormativo. Hay un cuestionamiento subversivo de los discursos normativos en un contexto de resistencia (Álvaro \& Pelúcio, 2013).

Otras propuestas de la contemporaneidad que indagan nuevas estéticas disonantes de la hegemonía occidental blanca, es la propuesta del artista brasileño Glauco Ferreira (2012), quien trabaja ampliando la noción de performance para interactuar con el público. Para la exposición colectiva Arte, Género y Activismo (Novo Hamburgo, 2012), Ferreira ha configurado un performance que se apoya en un dispositivo. El dispositivo es un conjunto de adhesivos sobre la pared que muestra la imagen de mujeres afrodescendientes, indígenas y otras que parecen ser no-occidentales, por lo menos fenotípicamente. Mediante los adhesivos, el artista explica su propuesta y lanza una pregunta en el espacio donde es realizada la primera parte de su propuesta que es el espacio expositivo - la galería -: ¿Quién precisa del feminismo interseccional? Luego a seguir, esta cuestión debe ser respondida a 
través de un sitio electrónico construido por el artista para este fin, documentando esta etapa del proceso de acción. Ferreira usa una estética de lo queer, connotando visualidades interseccionales como etnia, clase y sexualidad.

Otro dispositivo que puede ser analizado es Estudios Jotos (2013). Se puede tener acceso a esa propuesta en la Internet y/o en redes sociales como el Facebook. El dispositivo pretende, entre otros objetivos, atravesar identidades dicotómicas de hombre/mujer, masculino/femenino, animal/humano, usando performances fotográficas $y$ videográficas, conectando $y$ comentando otros eventos artísticos y militantes, a partir del entrecruzamiento de lo queer y lo decolonial. Recordando y aclarando para fines metodológicos y conceptuales del presente artículo que lo queer emerge en lo decolonial, en la poeta chicana Gloria Anzaldúa (1987). Para la poeta, existe un placer en el transitar libremente entre lo femenino y lo masculino (1987). Anzaldúa descubre una violencia en la dicotomía que obliga a optar o por lo femenino o por lo masculino. Inclusive, Anzaldúa propone ir más allá de dos géneros, utilizando el concepto de frontera como un espacio indeterminado en un residuo emocional, dentro del campo de los afectos, en un estado constante de transición, donde lo prohibido, las personas abjectas, son sus habitantes (1987). Al estar optando por la frontera, Anzaldúa es pionera en percibir la articulación que existe entre identidad de género e identidad nacional, dadas sus experiencias en territorio estadunidense en su condición de chicana. Esa indeterminación fronteriza es la espacialidad significacional del performance, es donde el fallo performativo acontece.

Este tipo de dispositivos, como Estudios Jotos, son implementados en el espacio virtual y en una intensa actualización con realidades políticas, como una documenta-acción por la supervivencia y, en clara lucha contra el feminicidio. El concepto de feminicidio fue usado por primera vez por la antropóloga mexicana Marcela Lagarde, precisamente para explicar el genocidio contra mujeres en México en 1990. Para Lagarde, feminicidio es el genocidio sistemático que se produce contra 
mujeres, dadas las condiciones históricas que permiten la generación de prácticas sociales contra la integridad, la libertad y la vida de las mujeres $y$, en recurrencia de la violencia normalizada debido a la ausencia de políticas estatales, convirtiendo el feminicidio en un crimen de Estado (2008). De los 25 países con mayor número de feminicidios en el mundo, 14 de ellos son latinoamericanos. Durante la guerra civil en Guatemala, fueron asesinadas aproximadamente mil quinientas mujeres. En México, consta el asesinato de 400 mujeres. La justicia se ha ejercido apenas para 20 por ciento de esos casos, siendo que mil mujeres mexicanas permanecen desaparecidas.

Lo queer en el dispositivo de Estudios Jotos es un disparador de significados de alerta en lo que se refiere a la institucionalización de la violencia contra género. Opera anteponiéndose a la estética heteronormativa. Las fotografías expuestas muestran personas actuando en el espacio público, muchas veces participando en manifestaciones políticas, mostrando afectos entre cuerpos parcialmente desnudos, parecen ser mujeres, pero también parecen no ser más mujeres. Existe un carácter lúdico y hedonista. En esas documentaciones difícilmente pueden ser identificadas visualidades binarias. Lo queer emerge como una zona fronteriza indicando que el performance de la desidentificación es la clave para escapar del acoso, de la violencia y de la muerte.

El punto es que el performance, bajo estos términos, es una estética que configura un espacio anti-letal y donde la visualidad, se transforma en cautiverio.

Esa estética se desenvuelve en la cultura pública, pero se refracta en otros espacios y campos del conocimiento, en la medida en que el imaginario blanco y heterosexual está siendo desplazado mediante esos dispositivos tecnológicos que atraviesan los espacios electrónicos, sexuales, domésticos, científicos, educacionales y nacionales. 
Consideraciones finales

Puede ser visto, cómo las prácticas artísticas analizadas en diálogo con los estudios de género y otras teorías de la antropología, bajo una perspectiva queer y decolonial, contribuyen para la configuración de un lenguaje estético que permite el tránsito entre diferentes géneros o, inclusive, más allá de la idea de identidad, como concepto para la determinación de los cuerpos y de las sexualidades.

También, es posible evidenciar cómo en la noción de performance, en el arte, no pueden ser esperados resultados previsibles, posteriores al evento. No existen negociaciones. En los estudios de género el performance sí llega a ser previsible. En diversas áreas del conocimiento, la noción de performance ha estado siendo empleada como un espectáculo artístico, como una forma de negar los lenguajes del arte tradicionales y como una manera de abrir el área del arte formal rumbo a la interdisciplinaridad.

Con seguridad, ninguna cultura debe cargar con el crimen de la esclavitud y/o de la conquista, o de los problemas raciales y del tráfico sexual, en las Américas postcoloniales. Es en ese sentido que se propone rescatar determinadas prácticas artísticas, como formas de tensión, más que como zonas liminares para la continuidad de una vida racial y dicotómica. La idea de liminaridad puede corresponder a un modelo de pensamiento evolutivo, con todas las consecuencias que ello implica.

En esta investigación, se ha mostrado cómo el contexto geopolítico puede mudar la concepción del performance, sus estudios y sus fines estéticos. Las condiciones y contextos en las que se realizan los estudios en performance debieren ser situados. Sus articulaciones con las teorías del arte consideran solamente aspectos occidentales, cuando en realidad, lo que habita y gravita en las Américas, lleva el nombre de feminicidio, de genocidio, en circunstancias críticas que encuentran en el performance una forma de arte para la expresividad insurgente, guerrillera $y$ libertaria, de manera más amplia. 
En su génesis, el/la artista se constituye así mismo/a como su propio objeto de investigación. Tal vez, bajo una perspectiva queer y decolonial, y como resultado principal de esta investigación, pueda ser afirmado que el performance, al (des)ocluir los procesos de subjetivación, apunte para una vida libre en una dimensión (des)autorizada del arte.

Cabría preguntarse cómo ha sido construido el arte eurocentrista, blanco y heternormativo, bajo qué tipo de exclusiones y proyectos raciales se ha blanqueado la historia del arte que, con mucho, acompaña el colonialismo científico y estético y otras disciplinas afines a su campo, como la crítica, la estética universalista, la teoría, la pintura, el dibujo, el grabado, la arquitectura $y$, en nuestros días, el performance de lo heterosexual.

Las prácticas artísticas como las realizadas por Estudios Jotos discuten el paradigma de lo social de las ciencias humanas de la cultura occidental, atravesando las identidades no dicotómicas como chamán, al mismo tiempo en que complican este tipo de categorías.

A modo de conclusión, puede ser afirmado que vivimos en una violencia epistemológica, sexual, científica y occidental, como producto de una jerarquización sistémica por parte de perspectivas cisgéneras que desean colocarse en un nivel superior a perspectivas trans o queers, controlando, actualmente, la producción del conocimiento. No se está hablando de jerarquías de opresión, se está refiriendo a jerarquías políticas y geográficas, entre lenguas, entre lenguajes artísticos en las Américas.

Urge llevar a cabo una decolonización de conceptualizaciones para identificar el conflicto político, científico cultural. Solamente de esa manera, la (re)interpretación de los acontecimientos y de la producción de subjetividades en el dominio del arte puede contribuir para la transformación de la ciencia o de su desaparición - de la ciencia -, generando nuevas formas de producción de conocimiento. 


\section{Referencias bibliográficas}

Álvaro, Lucas; PelÚCIO, Larissa. Self-portrait: Mapplethorpe e o terrorismo anal na arte pós-moderna. III Seminário Internacional Enlaçando Sexualidades. 15 a 17 de Maio. Universidade do Estado da Bahia, Salvador, 2013.

ANZALDÚA, Gloria. La consciencia de la mestiza. Towards a new consciousness (1987). In: CONBOY, K. et all. Writing the body: female embodiment and feminist theory. New York, Columbia University Press, 1997, pp.233-247.

BlANCA, Rosa Maria. Art de perspectives queer et questions postcoloniales aux Amériques. Transoceanik. Anais. Universidade Federal de Santa Catarina. Florianópolis, 2013.

Arte a partir de uma perspectiva queer / Arte desde lo queer. Tese (Doutorado Interdisciplinar em Ciências Humanas) Universidade Federal de Santa Catarina, 2011.

BUTLER, Judith. El género en disputa: El feminismo y la subversión de la identidad. Barcelona, Paidós, 2010.

CARRILlO, Eugeni Porras. Consideraciones sobre neochamanismo y chamanismo huichol. Gazeta de Antropología, vol. 19, n 07, 2003.

DoR, Juliette. "Christine de Pizan: de la transsexualité au travestissement". In: LEDUC, Guyonne (Dir.) Travestissement féminin et liberté(s). Paris, L'Harmattan, 2006.

Estudios Jotos, 2013 [https://www.facebook.com/pages/EstudiosJotos/118464691539708?fref $=$ ts $]$.

GLUSBERG, Jorge. A arte da performance. São Paulo, Perspectiva, 2007.

GOlDBERG, RoseLee. A arte da peformance: Do futurismo ao presente. São Paulo, Martins Fontes, 2006.

Gómez-PeÑA, Guillermo. Colectivo la Pocha Nostra. En defensa del arte del performance. Horizontes Antropológicos, vol. 11, $\mathrm{n}^{\circ}$ 24, Porto Alegre, 2005, pp.199-226.

HeNRÍQUeZ, Patricia. Oralidad y escritura en el teatro indígena prehispánico. Estudios filológicos, nº 44, 2009, pp.81-92. 
LABRA, Daniela. Crítica: Bienal de Arte de São Paulo - A arte da reflexão. $O$ Globo. 7 de setembro de 2014. [http://oglobo.globo.com/cultura/artes-visuais/critica-bienal-de-arte-desao-pauloarte-da-reflexao-13856635]

LAGARDE, Marcela. Antropología, feminismo y política: violencia feminicida y derechos humanos de las mujeres. In: BULLEN, Margaret; DIEZ, Carmen (Coord.). Retos teóricos y nuevas prácticas. XI Congreso de Antropología de la FAAEE. Donostia, Ankulegi Antropologia Elkartea, 2008, pp.209-239.

LlAMAZARES, Ana María. Occidente herido: el potencial sanador del chamanismo en el mundo contemporáneo. Diversidad, vol. 4, n ${ }^{\circ} 7$, 2013, pp.67-104.

MARTínEZ, José Luis. Música e representação no Kabuki, teatro anticonformista. Em pauta, vol. 15, n 24, 2004, pp.61-88.

MELIM, Regina. A fotografia como documento primário e performance nas artes visuais. Crítica Cultural, vol. 3, $\mathrm{n}^{\circ} 2,2008$. [http://www.portaldeperiodicos.unisul.br/index.php/Critica_Cultural/ar ticle/view/123/134]

MigNOLO, Walter D. La colonialidad a lo largo y a lo ancho: el hemisferio occidental en el horizonte colonial de la modernidad. In: LANDER, Edgardo (Comp.). La colonialidad del saber: Eurocentrismo y ciencias sociales. Perspectivas latinoamericanas. Buenos Aires, CLACSO, 2003.

NEGRISOLLI, Douglas. O corpo do performer nas artes visuais. Trama interdisciplinar, vol. 3, $\mathrm{n}^{\circ}$ 2, 2012, pp.147-154.

NERI, Christiane Soares Carneiro. Feminismo na Idade Média: conhecendo a cidade das damas. Revista Gênero \& Direito, $\mathrm{n}^{\circ} 1$, 2013, pp.68-85.

PIZAN, Cristina. La ciudad de las damas. Madrid, Siruela, 1405/2000.

PHELAN, Peggy. Unmarked: the politics of performance. London \& New York, Routledge, 1993.

Rocco, Patricia. Performing female artistic identity: Lavinia Fontana, Elisabetta Sirani and the allegorical self-portrait in sixteenth and seventeenth-century Bologna. Montreal, McGill University, Art History and Communication Studies, 2006. 
460 Performance: entre el arte, la identidad, la vida y la muerte

SALADIN D'ANGLURE, Bernard. Etre et renaître inuit, homme, femme ou chamane. Paris, Gallimard, 2006. 